\title{
SECOND WIND PHENOMENON IN CHRONIC CORONARY HEART DISEASE
}

\author{
BY \\ HAROLD N. SEGALL \\ From the Departments of Medicine, Montreal General and Jewish General Hospitals, and McGill University, \\ Montreal, Canada \\ Received November 24, 1964
}

"I have frequently when in company, borne the pain, and continued my pace without indulging it; at which time it has lasted from five to perhaps ten minutes and then gone off, as well as I can recollect, rather suddenly, as it came on than lessening gradually." This quotation from a letter written in 1772 to Heberden (1785) by a man who signed himself "Unknown" brought the second wind phenomenon to the attention of the great physician who first described the symptom complex which he named angina pectoris (Segall, 1945). Wenckebach (1924) spoke of this feature of cardiac pain, calling it the second wind phenomenon. A review of textbooks which deal with coronary artery disease published in the past 100 years shows that there has been little interest in the phenomenon, in fact it is rarely mentioned, let alone discussed in terms of the possible mechanism involved. Wenckebach's article on angina pectoris which I read in 1926 made me aware of it. The general impression gained from having listened to the story of symptoms in over 5000 cases of coronary artery disease leads me to estimate that not more than one in a hundred such patients volunteer the story of the second wind phenomenon and that it can be elicited by appropriate interrogation in about 4 per cent. A useful question of the indirect type is: "Have you ever experienced getting your second wind in sports like running or swimming?" An affirmative reply may be followed by the remark that the tightness in the chest, shortness of breath, or the sense of blocking in the chest for which the patient consults the physician bear a close resemblance to the sensations felt with the second wind phenomenon during sports. In most instances the physician must put the question more directly: "Have you noticed anything similar in connexion with the chest discomfort you experience in walking?" A negative reply then calls for spelling out the sequence of events: "You begin walking, and presently after two or three minutes the chest discomfort comes on and causes you to stop to rest. After a few minutes you get relief and you begin to walk again. How long can you walk before the discomfort reappears?" This is not precisely the same sequence of events as in the second wind phenomenon of a runner who continues running in spite of the discomfort until it disappears, but it is very closely related. Indeed, some patients can report both types of experience. Human nature does not change radically. Now, as in 1772, patients report that when walking in the company of others, wishing to avoid drawing attention to their disability, they continue to walk in spite of the pain until it disappears, precisely as patient "Unknown" did in Heberden's time. Such patients tend to benefit almost invariably from the prophylactic use of nitroglycerin which prevents the occurrence of the pain as they walk. Because the pattern of symptoms, which includes the second wind phenomenon, does not occur in relation to pain due to conditions that enter into the differential diagnosis of angina pectoris, it plays a minor role in confirming the diagnosis of coronary artery disease.

Following are clinical profiles of five patients who experienced second wind phenomenon related to the cardiac pain (angina pectoris) of chronic artery disease (Segall, 1950). 


\section{CASE REPORTS}

Case 1. Mr. A. L. (HNS2167), born 1893. First symptoms of impaired coronary circulation occurred at the age of 39 in November 1932 while walking outdoors; felt like a lump of food stuck under the midsternum causing a sense of pressure towards the epigastrium with vague aching in the left upper arm and the cheekbones. He had to stop in his tracks until rest gave relief in a few minutes. Carrying a parcel or hurrying tended to bring the pain on more readily. In December 1932, the electrocardiogram revealed no abnormalities. Nitroglycerin was prescribed to be taken prophylactically or for relief of pain. In January 1940 , he reported that he had enjoyed good health until July 1939, when he began to have a sense of pressure behind the sternum on walking for a few minutes on level ground, promptly relieved by stopping to rest for a few moments; then he could walk comfortably for an hour or longer. In January 1940, electrocardiograms recorded before, during, and after pain elicited by exercise revealed signs of myocardial ischæmia. In the next seven weeks he developed recurrences of cardiac pain of varying duration and intensity. Electrocardiograms recorded during pain presented striking signs of acute myocardial ischæmia. Serial electrocardiograms revealed evidence of anterior wall infarction in the last week of January 1940, and records made in the second week of February showed signs suggestive of posterior wall infarction. On the night of February 16 to 17, 1940, a severe attack of pain was associated with profound shock, during. which the patient remained alert and apprehensive. He died suddenly at 2 a.m. The clinical diagnosis included both stenosis and occlusion of coronary arteries with myocardial infarction involving the anterior and posterior walls.

Necropsy revealed tortuous firm coronary arteries with normal orifices in the aortic wall. The circumflex branch of the left coronary artery showed marked calcification and hyalinization with great narrowing of the lumen which was reduced in many places to a minute aperture that was eccentric. The right coronary artery revealed similar sclerosis and narrowing. Multiple transverse sections failed to reveal any point of arterial occlusion. The myocardium showed evidence of infarction involving the posterior and lateral walls of the left ventricle as well as the distal portion of the interventricular septum. This man who died at the age of 47 had second wind phenomenon related to chronic coronary artery disease six months before his death. Although the course of his illness in the last two months of life was carefully observed and recorded it has not been possible to correlate the necropsy observations with the variations in duration and intensity of pain. It seems unlikely that what happened in November 1932, or in July 1939, could be accounted for with any degree of precision by what was found at necropsy in February of 1940 when dealing with cardiac pain related to coronary circulation.

Case 2. (HNS43404). In September 1955, while playing golf, this man of 55 first noticed the pain of chronic coronary artery disease (angina pectoris) as a sense of pressure and tightness under the lower third of the sternum. It persisted until he stopped to rest at the second tee. It recurred while walking to the third tee and cleared as he rested there. Then, he played the remainder of 18 or 36 holes free from any discomfort. In November 1955, he had acute coronary artery disease with anterior wall myocardial infarction. This. was a mild illness and during convalescence in Florida in February 1956, he was able to walk a mile without any discomfort. On his return to the winter climate of Montreal, he found that after walking a minute or two outdoors, he experienced a sense of pressure under the upper third of the sternum. This was relieved within a few seconds if he stopped to rest. It would recur after walking again for three to five minutes. He also had the pain when emotionally tense during a business conference. On such occasions, nitroglycerin relieved the pain within half a minute. In the spring of 1956, he was able to play nine holes of golf without any pain. In the autumn of 1956, cardiac pain on walking recurred. In September and October of 1956, he was found to have thyroiditis which responded to x-ray treatment, for he recovered his general sense of well-being and also became free of cardiac pain on walking. In the spring and summer of 1957, he played a round of golf without any discomfort. In the winter of 1958, he had pain on walking soon after a meal but none when his stomach was empty. In the summer of 1958, he reported that he could walk for five miles around a golf course in a relaxed mood without discomfort, but if he hurried on his way to the bus in going to work, walking for only five minutes brought on the discomfort in the chest which was relieved as soon as he sat down in the bus. In the winter of 1959, the pain appeared after walking only 100 yards or so, was relieved by rest for a minute, and then recurred after walking for another 50 or 100 yards. He had no second wind phenomenon at this time. He disliked using nitroglycerin. He was encouraged to use it prophylactically in the form of nitrostabilin. In the summer of 1959, after retiring from his executive position, he played golf without any discomfort. He remained in this state until the autumn of 1962 when he began to feel a sense of pressure under the middle of the sternum on walking outdoors for a few minutes. 
Rest gave relief within a few moments and then he could walk a long distance without recurrence. In December 1963, retrosternal pain began to come on frequently, not only after about two minutes of walking but with the slight exertion of lifting a garage door or drying himself after taking a shower. This was considered evidence of subacute coronary artery disease (pre-infarction syndrome) which was treated with a convalescent régime at home and anticoagulants. After three weeks of rest, he resumed his usual activities. In the next three months, he had three episodes of pain which woke him from sleep and which were promptly relieved by nitroglycerin. In October of 1964, he reported that walking about 300 yards brought on the retrosternal pain which was promptly relieved by nitrostabilin in a minute or so and then he could walk for an hour or more without any recurrence of pain. Thus, the second wind phenomenon returned. The electrocardiogram remained unchanged from December 1955, to March 1964, but in October 1964, slight inversion of T in AVL and in V1 were new features suggestive of some ventricular myocardial fibrosis involving the left lateral wall of the ventricle. The lesion probably occurred at some time in the interval between March and October. There was no evidence of any acute disease.

Case 3. (HNS18379). In 1943, this man experienced progressive difficulty in climbing stairs or hills because of shortness of breath and palpitation. In February 1944, he walked up a steep hill carrying a roll of maps which weighed $20 \mathrm{lb}$. $(9 \mathrm{~kg}$.), in spite of increasing shortness of breath, until he reached the top where he felt a "blackout"; he could not see before him. He managed to reach a chair, did not lose consciousness, but felt severe thumping and throbbing in his chest and head and weakness in the knees. Perspiring profusely he rested for ten minutes and then was able to resume walking. The clinical examination revealed no evidence of cardiovascular or pulmonary disease, but he was obese, weighed $226 \mathrm{lb}$. (102 kg.), height $5 \mathrm{ft} .9$ in. $(175 \mathrm{~cm}$.). His symptoms were attributed to poor physical condition related to obesity and lack of exercise. The symptoms fit the description of what is felt during the second wind phenomenon by athletes. In 1959, he experienced pain across the upper part of the chest with occasional aching in the right arm and, rarely, twinges in the left arm while walking outdoors especially when hurrying. The electrocardiogram revealed abnormalities indicative of myocardial fibrosis in the anterior ventricular wall. Once the pain was relieved by stopping to rest, he was able to walk a long distance without any discomfort. In the autumn of 1959, this second wind phenomenon disappeared and he had to stop five times while walking for about ten minutes in the course of his business activities. However, on the golf course he did experience a second wind phenomenon. In November 1961, he reported that the symptoms had changed slightly. The pain did not recur after the first episode early in a walk if he slowed his pace, but there was recurrence if he resumed his walk at a normal pace. In July 1964, he reported that he was entirely free from pain on walking during the course of the day or while playing 18 holes of golf unless he walked soon after a meal, and then the pain came on and a second wind phenomenon occurred.

Case 4. (HNS48171). In the summer of 1956, at the age of 45, this man was walking during a fishing trip, when he developed tightness or pressure across the upper part of the chest. He sat down for a few minutes until it cleared away. Then he resumed walking and had no recurrence of the pain during the remainder of a long walk. In the summer of 1957, the pain recurred while playing golf. It developed as he walked up the incline on the first fairway and persisted until he reached the third tee, when it disappeared, and he was able to play the remainder of the 18 holes without any discomfort. In November of that year, walking hurriedly to meet a friend, he had to stop five times in the course of a 15-minute walk to get relief from the pain. A few days later, he consulted a physician who diagnosed acute coronary artery disease. He returned to work in March of 1958 but had frequent recurrences of pain which were promptly relieved by nitroglycerin. Second wind phenomenon was not a feature during this time. He became free enough from pain to resume work as a manager of a bank branch in January 1959. Between 1961 and 1964 he was free of pain. In November 1964, intermittent claudication affected his left leg, and when this pain became very intense it tended to initiate retrosternal pain which disappeared as soon as he stopped to rest, while the calf pain took longer to disappear. Before the onset of intermittent claudication, he was able to walk an hour or longer or to play 18 holes of golf without any chest discomfort.

Case 5. (HNS44891). At the age of 42, on October 24, 1954, this man swam one and one-half lengths of a pool, and then felt a painful pressure sensation which began at the level of the fourth dorsal vertebra and radiated anteriorly directly to the upper portion of the sternum. He rested for a minute or so and it disappeared. That evening, after walking 100 yards from his house, the same pain recurred. It disappeared in a minute or so. He walked back at a slower pace and had no pain. Subsequently, he noticed that walking hurriedly outdoors was the only trigger mechanism that initiated the pain. In December 1954, on one occasion about two hours after the midday meal, he walked a distance which normally required five minutes but occupied 20 minutes because of recurrences of pain at about every 20 paces causing him 
to rest for half a minute or so until it was relieved. Subsequently, until December 1962, he continued to take regular exercise in the form of walking, swimming, and golfing and observed that he developed chest pain during the first few minutes of exercise, and rest alone or together with nitroglycerin brought relief in less than a minute, after which he was able to continue swimming or walking for half an hour or more without any discomfort. In December 1962, he developed acute coronary artery disease (Segall, 1950) with posterior wall infarction. During the summer of 1963, after he had had a long convalescence, he nevertheless again experienced pain after the first two or three minutes of walking outdoors. Stopping to rest gave relief in about 30 seconds to a minute, then he was able to walk for half an hour or more without any discomfort. Frequently, if he took a pill of nitroglycerin before beginning to play golf he would be free of pain entirely. At other times, the pill of nitroglycerin did not prevent the recurrence of pain within the first ten minutes of walking.

\section{DisCUSSION}

In his book on the Physiology of Muscular Exercise, Bainbridge (1931) devotes two pages to the subject of second wind. These may be summarized in the following quotation: "There appears. to be no doubt of the fact that the onset of second wind is associated with a number of adjustments, including oxygen intake, carbon dioxide output, the pulse rate and often the onset of sweating ... It represents establishment of a steady state in which numerous variables are concerned." He does not mention adaptation of the coronary circulation. The possibility that this factor plays a role in second wind phenomenon came to my mind as a result of two personal experiences. In the spring. of 1924, when I was serving as Resident in Cardiology at the Massachusetts General Hospital, Dr. R. G. Vance, the Resident in the Department of Radiology, engaged my interest in an experiment which he wished to perform. In search of a method for slowing the flow of barium through the second portion of the duodenum, he conceived the idea of lowering a collapsed balloon with a piece of lead shot in it at the end of a duodenal tube into the third portion of the duodenum. He would then have the subject swallow a barium mixture and before it reached the duodenum he would block its path by blowing up the balloon and thus make for good visualization of the entire duodenum. I undertook to be his subject; on one occasion, as the balloon was moving down the œsophagus under fluoroscopic guidance, I asked him to stop it in the vicinity of my aorta and blow it up so as to see the effect of this mechanical disturbance on the nerve supply of that region. The effect was that of producing an excruciatingly intense squeezing pressure pain under the entire front of the chest maximally behind the manubrium sterni with concommitant tingling pain in the shoulders, arms, and lower jaw and profuse sweating. I allowed the pain to persist long enough to describe it with precision and then, once the balloon was deflated the pain quickly diminished in intensity and disappeared entirely within less than two minutes. This impressed me as similar to cardiac pain of the kind described by people who suffer from impairment in coronary circulation. The next event which provided me with an occasion to experience a similar pain occurred in the winter of 1927. During a snowstorm, I was driving my car along a narrow country road and, lacking adequate markings, I steered the car into the ditch and stopped it when the two left wheels were in the ditch and the two right wheels were still on the road. This called for assistance by a farmer with a pair of horses. The farmhouse was about 400 yards away. I began to walk through the knee-deep snow towards the farmhouse in a mood of considerable hurry and urgency. When I had gone perhaps 100 feet, I developed severe retrosternal pain with maximal intensity about the middle of the sternum and consciousness of difficulty in breathing, which caused me to stop because I felt I could not move another inch until this pain cleared. After resting for about three minutes, I began to walk again, but this time in a leisurely mood and relatively slowly. I reached the farmhouse without any recurrence of symptoms.

At that time, my tentative explanation for the second wind phenomenon was that I probably did have coronary arteries that were not quick enough to adapt to the task I had imposed on them. This could be due to arteriosclerotic stenosis in a man of 29. (In 1926, I had seen a case of acute coronary occlusion with myocardial infarction in a medical student aged 24 . Neither then, nor in the next 37 years have I had symptoms or shown signs of coronary artery disease.) The other, and 
more optimistic, explanation was that a reflex regulation of coronary circulation in a person not in good athletic condition might make for inadequate vasodilatation, whereas in a well-trained athlete, the reflexes would have been so conditioned that the subject could perform at maximum capacity without discomfort. The mechanism of the reflex that I had in mind was as follows. It had been established by Anrep and Segall (1926) that the vagus nerve maintained a constrictor tone of the coronary arteries, and the sympathetic nerve supply to these arteries when stimulated caused vasodilatation. Thus, reflexes from lungs or skeletal muscles would inhibit the vagus effect and stimulate the sympathetic so as to produce an abundant coronary circulation. This reflex would be in the same category as the Bainbridge reflex by virtue of which the heart rate increases when the right atrium is distended by an increase in venous return, which occurs with physical exercise. This reflex is at least one significant factor in producing the tachycardia associated with physical exertion. Assuming that there is a significant mechanism that functions to adapt coronary circulation to the needs of the heart by reflex action, this mechanism probably plays a role in adjustments that occur when stenosis of a coronary artery impairs blood flow. The tendency would be for reflex action to contribute something to a compensatory mechanism that would provide adequate flow of blood to the affected area of myocardium so as to prevent the occurrence of pain. This pattern of thinking supplies a tentative answer to the question, Why is it that in an important number of cases the necropsy reveals stenosis of coronary arteries, yet the patient had not suffered from cardiac pain? Of course, additional factors, namely, intercoronary anastomoses must play an important role in the compensatory mechanism. It becomes only a matter of degree whether a subject develops second wind phenomenon when he performs exercise analogous to walking in deep snow hurriedly in a state of urgency or walking on level ground at a normal pace in an unhurried mood. In the latter case, the pain would result from the inadequacy of coronary blood supply due to stenosis of coronary arteries, and the second wind phenomenon in such circumstances would be related to the reflex mechanism mentioned above.

In 1927, a patient (HNS20) who sought to "cure" himself of "angina pectoris" by regular exercise in a gymnasium and who succeeded in becoming able to walk outdoors without pain led me to prescribe regular exercise, usually walking or swimming, in such cases. In some instances this seemed to have the desired effect. In others, cardiac pain and the second wind phenomenon persisted though exercise did improve the general physical condition. In the majority, the tendency for cardiac pain to occur was not significantly affected.

In his Lane Lectures, Anrep (1936) discussed the relation between angina pectoris and reflex regulation of the coronary circulation. He mentioned a type of angina pectoris in which exercise tolerance is increased after the subject recovers from the cardiac pain. This probably represents second wind phenomenon as observed by the patient "Unknown" and by the five patients described above. In this connexion he stated: "At least in some cases of angina pectoris there still remains a certain adaptation of coronary blood flow to the requirements of the heart, but this adaptation is slow in appearance and follows rather than coincides with the period of exercise." In his studies of the coronary circulation using the heart-lung-brain preparation, he found that "the sympathetic vasodilator fibres can be stimulated reflexly. Thus, electrical stimulation applied to various sensory nerves, such as the sciatic, leads to augmentation of the coronary blood flow which disappears after extirpation of the stellate ganglia." Thus, one may envisage reflexes from skeletal muscles affecting coronary circulation.

In the studies that were made by Anrep and his associates on the heart-lung-brain preparation, coronary circulation was measured rather crudely by collecting blood from the coronary sinus. More recently, Gregg $(1950,1963)$ and others have applied more direct methods of measuring coronary arterial flow using electronic flowmeters. Gregg demonstrated that during excitement or during electric stimulation of the cardiac sympathetic nerve, the left coronary bed underwent massive dilatation, so that the left ventricle was able to increase its coronary circulation as the result of the combined effect of an increase in number of heart beats and greatly increased blood flow and oxygen usage per heart beat. These studies suggest that the nerve supply to the heart may play an 
important part in regulating coronary circulation. The precise manner in which it functions in relation to the second wind phenomenon requires investigation. The function of the central and reflex regulation of coronary circulation in the human being with impaired coronary circulation remains a matter of conjecture in the absence of human experimental data. However, this element should not be ignored in calculating the total function of the heart in normal subjects and those with coronary artery disease.

Recently, MacAlpin, Kattus, and Winfield (1964) have studied the second wind phenomenon during systematically controlled exercise, walking on a treadmill. Using the technique of angiography, they demonstrated coronary collateral circulation in many of these cases. In three patients, regular daily exercise of walking as much as 4 miles a day strikingly improved treadmill performance. Studies of this kind will surely advance our knowledge of the circulatory dynamics both in normal healthy people and in those with coronary artery disease who experience second wind phenomenon.

\section{SUMMARY}

The second wind phenomenon occurs occasionally in patients who have cardiac pain associated with chronic coronary artery disease. The physiology of second wind phenomenon as it occurs in normal subjects engaged in athletics is not yet clearly understood. Its occurrence in relation to cardiac pain draws attention to the possibility that adaptation of the coronary circulation by reflex regulation plays an important role in its dynamics. The pattern of symptoms in five cases of chronic coronary artery disease and in one normal subject is described.

\section{REFERENCES}

Anrep, G. V. (1936). Lane medical lectures: studies in cardiovascular regulation. Stanf. Univ. Publ. med. Sci., 3, 199. and Segall, H. N. (1926). The regulation of the coronary circulation. Heart, 13, 239.

Bainbridge, F. A. (1931). The Physiology of Muscular Exercise, 3rd ed., p. 188. Longmans, Green, London, New York, Toronto.

Gregg, D. E. (1950). Coronary Circulation in Health and Disease, p. 131. Lea and Febiger, Philadelphia. (1963). The George E. Brown memorial lecture: Physiology of the coronary circulation. Circulation, 27, 1128.

Heberden, W. (1785). A letter to Dr. Heberden, concerning the angina pectoris; and Dr. Heberden's account of the dissection of one, who had been troubled with that disorder. Med. Trans. roy. Coll. Phys., London, 3, 1.

MacAlpin, R. N., Kattus, A. A., and Winfield, M. E. (1964). Abstract of paper delivered at thirty-seventh scientific sessions of the American Heart Association. Evidence for adaptive mechanisms in angina pectoris leading to improvement in exercise capacity (P). Circulation, 30, Suppl. 3, p. 119.

Segall, H. N. (1945). The first clinico-pathological case history of angina pectoris; self-diagnosis by anonymous physician; autopsy by John Hunter; reported by William Heberden in 1772. Bull. Hist. Med., 18, 102.

(1950). The principal patterns of cardiac pain related to arteriosclerotic coronary artery disease. Canad. med. Ass. J., 62, 341.

Wenckebach, K. F. (1924). Angina pectoris and the possibilities of its surgical relief. Brit. med. J., 1, 809. 